\title{
Glass Transition as the Rheological Inverse of Gelation
}

\author{
H. Henning Winter* \\ Department of Chemical Engineering and Department of Polymer Science and Engineering, University of Massachusetts, Amherst, \\ Massachusetts 01003, United States
}

\begin{abstract}
This is a comparative study in search for common patterns in the relaxation dynamics of amorphous materials in the approach of a liquid-to-solid transition from the liquid side. Observations with two representative materials provide guidance for the study. The first material, a concentrated colloidal suspension, represents the glass transition. The second material is a cross-linking polymer far above its glass transition; it represents gelation. The entire study is founded in Boltzmann's constitutive equation of linear viscoelasticity; the stress is caused by a wide range of relaxation modes where, as argued here, fast modes dominate gelation and slow modes dominate the glass transition. For both classes

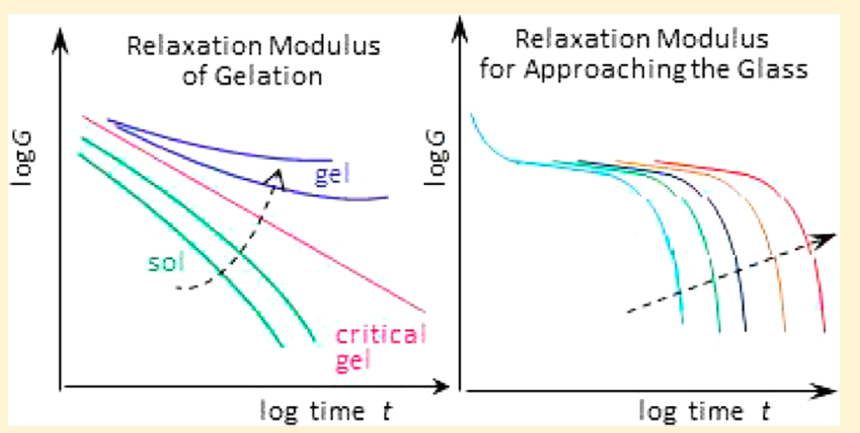
of amorphous materials, the relaxation time spectrum broadens and adopts powerlaw format, but the powerlaw exponent is positive for the glass transition and negative for gelation, i.e. the relaxation patterns of gelling fluids and glass formers are inverse near the transition. Several examples are shown for each class of materials in order to test the proposed transition behavior for glasses (colloidal and molecular) on the one side and chemical/physical gels on the other. Among several results, this experimental study provides a decisive criterion that distinguishes the glass transition from gelation. It also shows a relation between the zero shear viscosity and the diverging longest relaxation time for both materials.
\end{abstract}

\section{INTRODUCTION}

Recent experimental and theoretical advances allow a combined look at the rheology of the glass transition as compared to gelation, both being distinct phenomena of nature that slow the dynamics and eventually arrest the internal motion of either material's constituents. The transition may be caused by a wide range of different connectivity mechanisms while rheological consequences share similar features macroscopically. ${ }^{1-7}$ This raises the question about the existence of a common constitutive model that provides a quantitative framework for their description. The rheological understanding of the solidification behavior of amorphous materials is a major scientific challenge, but it also is of practical importance since many industrial products and processes rely on the control of rheological properties near the transition from liquid to solid.

To begin with, two model materials were chosen for this comparative experimental study, one for each material class. Gels are represented by a chemically cross-linking polymer that is approaching its gel point, but remains far above its glass transition temperature. Soft glasses are represented by a colloidal suspension near the glass transition due to high particle loading; a short-range repulsive particle-particle potential prevents gelation and slight polydispersity suppresses crystallization. The study draws from a range of published viscoelasticity data for the two representative model materials. The analysis of these data results in a relaxation time spectrum, which then gets tested on other gels and glasses near the liquidto-solid transition.
At this state of understanding we purposely set a narrow focus for this study, which is restricted to the liquid-to-solid transition from the liquid side (LSTLS). The selected model materials are clearly governed by either gelation or by the glass transition (no competing states). Excluded is the dynamics of the solid state, which would be a large topic by itself. In particular, glassy materials beyond LSTLS would require extensive attention because of their out-of-equilibrium metastable states. The scope of this experimental study is purposely restricted to experimental rheology although it should be noted that other experimental methods can help to distinguish gelation from the glass transition. ${ }^{8}$

The constitutive modeling in this study is founded in Boltzmann's equation ${ }^{9}$ for the stress $\sigma(t)$ response to a strain rate history, $\dot{\gamma}\left(t^{\prime}\right)$ for $-\infty<t^{\prime}<t$

$$
\sigma\left(t, \varepsilon_{i}\right)=\int_{-\infty}^{t} \mathrm{~d} t^{\prime} G\left(t-t^{\prime}, \varepsilon_{i}\right) \dot{\gamma}\left(t^{\prime}\right)
$$

Parameter $\varepsilon_{i}$ defines the distance from the transition as will be explained below. The relaxation modulus $G(t)$ is a monotonically decaying function in time since, for liquids, the most recent past history has the most influence on the stress. Boltzmann showed, by means of the linear superposition principle, that a single (!) rheological material function completely defines the linear viscoelastic behavior of a stable,

Received: January 14, 2013

Revised: February 25, 2013 
isochoric material in shear, extension, or any mixed flow, transient or steady. No other material function is needed (assuming constant density) beyond $G(t)$. However, $G(t)$ can be expressed in other equivalent ways, for instance as weighted time integral over all relaxation modes for $\tau \leq \tau_{\max }$

$$
\begin{aligned}
& G\left(t, \varepsilon_{i}\right)=G_{e}+\int_{0}^{\tau_{\max }} \frac{\mathrm{d} \tau}{\tau} H\left(\tau, \varepsilon_{i}\right) \mathrm{e}^{-t / \tau}, \\
& \text { with } G_{e}=0 \text { for liquids }
\end{aligned}
$$

Equation 2 defines the relaxation time spectrum $H(\tau)$. The upper limit of the relaxation time spectrum, with a longest relaxation time $\tau_{\max }$ belongs to the largest connected material region that can still relax. In general, there is no preference for using either $H(\tau)$ or $G(t)$ to express the viscoelasticity of a material. This study, however, finds that $H(\tau)$ captures experimental LSTLS data in a simpler mathematical format so that preference will be given to $H(\tau)$.

The modulus and the relaxation time spectrum in Boltzmann's constitutive equation is written here as a function of the distance from the transition, $\varepsilon$, with $\varepsilon=0$ at the transition point. Vastly different connectivity mechanisms in gels and glasses require an appropriate definition for the separation parameter $\varepsilon$ which naturally varies from material to material. In the close vicinity of the chemical gel point of a crosslinking polymer, the separation parameter is defined as the distance from the critical reaction conversion, $\varepsilon=p_{c}-p$, with $p_{c}$ being the bond probability at the gel point. Less clear is the definition of $\varepsilon$ for physical gels, which may connect with a wide range of different clustering mechanisms; ${ }^{10} \varepsilon$ needs to be reconsidered from case to case. Colloidal glass formers are subject of a separation parameter $\varepsilon=\left|\phi-\phi_{c}\right| / \phi_{c}$, for which the critical volume fraction of particles, $\phi_{c}$, varies with experimental conditions ${ }^{11-14}$ because of trapped nonequilibrium conditions of the sample structure, and it depends on particle size. The actual value of $\phi_{c}$ for a specific experiment might be lower than theory would predict. ${ }^{15}$ Colloidal dynamics will be compared to the dynamics of temperature-dependent, molecular glass formers that change their internal connectivity with $\varepsilon=\left|T-T_{\mathrm{g}}\right| / T_{\mathrm{g}}$ involving a glass transition temperature, $T_{\mathrm{g}}$, that typically depends on the cooling rate in the approach of LSTLS.

This study will proceed directly to the determination of linear viscoelasticity for gelation and the glass transition in order to compare the two phenomena. Following Boltzmann, all we need is $G\left(t, \varepsilon_{i}\right)$ or $H\left(t, \varepsilon_{i}\right)$ of model gels and model glassy materials at discrete, stable states $\varepsilon_{1}, \varepsilon_{2}, \varepsilon_{3}$,... near LSTLS. The longest relaxation time $\tau_{\max }\left(\varepsilon_{i}\right)$ diverges in the approach of LSTLS, $\varepsilon_{i} \rightarrow 0$. It will be shown that both material groups share the same powerlaw relaxation time spectrum,

$$
H\left(\tau, \varepsilon_{i}\right)=H_{0}\left(\frac{\tau}{\tau_{\max }\left(\varepsilon_{i}\right)}\right)^{n}, \quad \text { for } \tau<\tau_{\max }
$$

except that the exponent is positive $(n \geq 0)$ for the glass transition and negative $(-1<n<0)$ for gelation. In this framework, gelation is viewed as the rheological inverse of the glass transition. This will be derived in the following and tested on several amorphous materials.

Determination of the Relaxation Time Spectrum. While many studies of LSTLS focus on the longest relaxation time (which superficially looks quite similar for gelation and the glass transition), this study focuses on the broad distribution of relaxation times and the associated pattern since these express the difference between gelation and the glass transition most clearly. The relaxation time spectrum $H(\tau)$ is a macroscopic expression of small-scale structural rearrangements in time. Relaxation processes at short $\tau$ refer to small-scale rearrangements in a test material. Correspondingly, long-time relaxation depends on large-scale rearrangement processes of material constituents. $H(\tau)$ may adopt any functional shape depending whether short or long-time structural rearrangements dominate the stress.

$G(t)$ and $H(\tau)$ can be effectively determined by measuring dynamic moduli,

$$
\begin{gathered}
G^{\prime}(\omega)=G_{e}+\int_{0}^{\tau_{\max }} \frac{\mathrm{d} \tau}{\tau} H(\tau) \frac{(\omega \tau)^{2}}{1+(\omega \tau)^{2}} ; \\
G^{\prime \prime}(\omega)=\int_{0}^{\tau_{\max }} \frac{\mathrm{d} \tau}{\tau} H(\tau) \frac{(\omega \tau)}{1+(\omega \tau)^{2}}
\end{gathered}
$$

in small-amplitude oscillatory shear (SAOS) and then extracting $H(\tau)$ or $G(t)$. This is the approach taken here. Equations 4 derive from eq 1 when inserting the SAOS shear rate. ${ }^{16}$ The data analysis in this study uses the parsimonious modeling approach, which attempts an unbiased fit function for $H(\tau)$ that optimally represents the data with the smallest parameter set. The discrete spectrum is then converted into a continuous spectrum. ${ }^{17,18}$

Rheological complexity arises from variations in $\varepsilon$ near LSTLS and from the corresponding structural changes. The transient structure near LSTLS conflicts with Boltzmann's requirement of a stable material structure during the contributing strain rate history. Material stability is a prerequisite for eq 1 which would be invalid for time-dependent $\varepsilon(t) .{ }^{19}$ Because of this limitation, rheological experiments will have to rely on iso-evolution conditions, i.e. stabilized samples at constant distance from the transition, $\varepsilon_{i}=$ const., or quasistable conditions for which $\varepsilon$-changes are negligibly small during the taking of a data point. Throughout this study, when expressing viscoelasticity in terms of $G\left(t, \varepsilon_{i}\right)$ or $H\left(t, \varepsilon_{i}\right)$, the separation from LSTLS, $\varepsilon$, will not be treated as a variable but as a discrete, independent parameter that defines the state of advancement in gelation or the glass transition. Also, stress or strain induced effects need to be avoided at this level of understanding; this has been achieved by measuring the linear viscoelastic functions at sufficiently small strain.

For linear viscoelastic characterization of samples with slowly changing $\varepsilon(t)$, Fourier transform mechanical spectroscopy ${ }^{20,21}$ and time-resolved rheometry ${ }^{19}$ are suitable. Data points are deemed acceptable as long as both mutation numbers, ${ }^{19}$

$$
N^{\prime}{ }_{m u}=\frac{2 \pi}{\omega G^{\prime}} \frac{\partial G^{\prime}}{\partial t} ; \quad N_{m u}^{\prime \prime}=\frac{2 \pi}{\omega G^{\prime \prime}} \frac{\partial G^{\prime \prime}}{\partial t}
$$

stay below a tolerable limit (typically $N^{\prime}{ }_{m u}<0.1$ while $N^{\prime \prime}{ }_{m u} \ll$ $N^{\prime}{ }_{m u}$ ). SAOS is a spectroscopic technique which reaches quasistable condition in about one sampling period. This is why $2 \pi$ / $\omega$ is chosen as reference time in the definition of the mutation numbers. Time-resolved rheometry allows the study of a material's response under slightly dynamic conditions.

Relaxation Time Spectrum of Gelation. In chemical gelation, covalent bonds connect molecules into a highly branched molecular structure with a molecular size distribution, which is still broad at intermediate reaction conversions near LSTLS. A chemically cross-linking material is ideally suited for the study of gelation as long as the bond probability can be controlled chemically and the glass transition if way outside the 
experimental region. The long levity of the chemical bonds is advantageous since it provides stability for the rheological study. Endlinking poydimethylsiloxane has successfully served as such a model material for rheological experiments, which led to the discovery of the powerlaw relaxation time spectrum at the gel point, $p=p_{\mathrm{c}}^{22-25}$

$$
H\left(\tau, \varepsilon_{i}\right)=H_{0}\left(\frac{\tau}{\tau_{0}}\right)^{n}, \quad \text { for } \tau>\tau_{0} \text { with }-1<n<0
$$

For performing the rheological experiments, the cross-linking reaction had either been stopped at discrete $\varepsilon_{i}$ near the gel point or cross-linking was slowed down to maintain low mutation number values. $\tau_{0}$ is a short relaxation time which characterizes the structural building block of the gel. ${ }^{26}$ The corresponding dynamic moduli for the above powerlaw spectrum with negative exponent, eq 6, can be expressed analytically

$$
\begin{aligned}
& G^{\prime}(\omega)=\frac{\pi H_{0}\left(\tau_{0} \omega\right)^{-n}}{2 \sin \left(-\frac{n \pi}{2}\right)} ; \quad G^{\prime \prime}(\omega)=\frac{\pi H_{0}\left(\tau_{0} \omega\right)^{-n}}{2 \cos \left(-\frac{n \pi}{2}\right)} ; \\
& \text { with }-1<n<0 \text { for } 0<\omega<1 / \tau_{0}
\end{aligned}
$$

The loss tangent becomes frequency independent, $\tan \delta=G^{\prime \prime} /$ $G^{\prime}=$ constant. The normalized loss angle equals the powerlaw exponent, $n=-2 \delta / \pi^{25}$

Near the gel point (finite $\varepsilon$ ), the spectrum is cut off at the largest relaxation time, $H=0$ for $\tau>\tau_{\max }$. Below $\tau_{\max }$ the relaxation time spectrum can be approximated as $H=\bar{H}_{0}(\tau)$ $\left.\tau_{\max }\right)^{n}$ for $\tau>\tau_{0}$. Friedrich et al. ${ }^{27}$ successfully used a truncated powerlaw spectrum to describe the relaxation near the chemical gel point.

Since its discovery in 1985, the powerlaw spectrum at the gel point has been found for a wide range of diverse chemical and physical gels. Recent examples are studies by Morris et al., ${ }^{28}$ Ghiringhelli et al., ${ }^{21}$ Aliaghaie et al., ${ }^{29}$ Aoki, ${ }^{30}$ Bonino et al., ${ }^{31}$ $\mathrm{Ng}$ et al., ${ }^{32}$ and Eberle et al. ${ }^{33}$

Instead of showing the 1985 data again, the viscoelastic gelation data of a polybutadiene (PB) of DeRosa et al. ${ }^{34,35}$ is analyzed in Figures 1 and 2. The data are typical for the chemical gelation of a cross-linking polymer. Both, $G^{\prime}$ and $G^{\prime \prime}$ grow with increasing extent of cross-linking (increasing reaction time, listed in Figure 2b). Three states are selected in Figure 1a, including the transition state which shows the powerlaw. The resulting loss angle, Figure $1 \mathrm{~b}$, decays and assumes a constant value for most of the data, including the lowest frequencies. The normalized loss angle, $2 \delta / \pi$, begins to tip down at the lowest frequencies when extending the measurements beyond the gel point. The dynamic moduli for the approach of LSTLS can be merged into a master curve, Figure $2 a$, as already shown by Adolf and Martin. ${ }^{36}$ However, while $G^{\prime}$ and $G^{\prime \prime}$ overlay nicely, the $\tan \delta$ curves suggest that the shift is not really satisfying. For this cross-linking PB, the powerlaw dynamics is only valid in a narrow window close to the chemical gel point. The magnitude of the horizontal shift (frequency shift) is a measure of the slowdown of the dynamics, Figure $2 b$.

Relaxation Time Spectrum of the Glass Transition. Concentrated colloidal suspensions of particles characterized by a sufficiently steep mutual repulsion may be treated as the most ideal model system for studying near-glass dynamics. $1,11,37-40$ Two pronounced relaxation processes, known as $\alpha$ - and $\beta$ relaxation, each with its distinct pattern of relaxation times and strengths, govern the near-glass dynamics of LSTLS. For

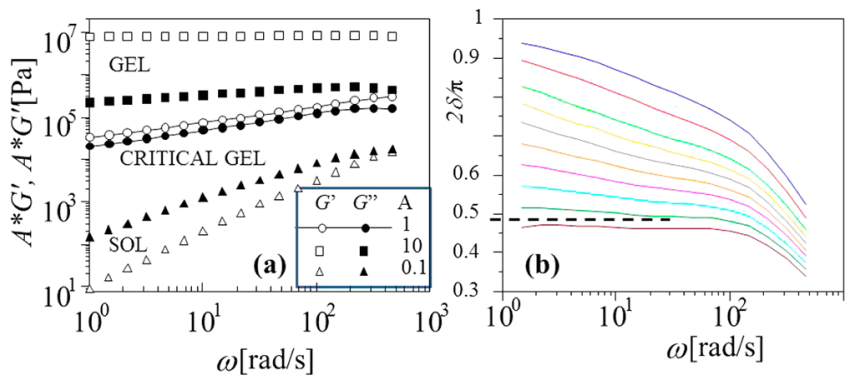

Figure 1. Data of DeRosa et al.; ${ }^{34,35}$ dynamic moduli and loss angle $\delta$ of chemical gelation at $T=28{ }^{\circ} \mathrm{C}$ of a polybutadiene $\left(M_{\mathrm{w}}=18100 \mathrm{~g} /\right.$ mol; $M_{\mathrm{w}} / M_{\mathrm{n}}=1.03 ; \%$ cis $=41 ; \%$ trans $=52 ; \%$ vinyl $=7.3 ; T_{\mathrm{g}}=-92$ ${ }^{\circ} \mathrm{C}$ ) with p-bis(dimethylsilyl) benzene as difunctional crossliker. (1a) $G^{\prime}(\omega)$ (open symbols) and $G^{\prime \prime}(\omega)$ (filled symbols) of three selected cross-linking states denoting the sol, the critical gel, and the gel. (1b) The normalized loss angle $\delta$ decreases at advancing reaction times 696s, 796s, 894s, 944s, 993s, 1042s, 1091s, 1142s, 1196s, and 1245s, which correspond to increasing chemical conversions as shown by DeRosa et al. ${ }^{35}$ The gel point is reached after $1196 \mathrm{~s}$. The critical gel expresses itself in parallel moduli (1a) and a flat $2 \delta / \pi(1 \mathrm{~b})$ at low frequencies. The property values at the gel point are $n=-0.43$ and $S=$ $9200 \mathrm{~Pa} \mathrm{~s}^{0.43}$.

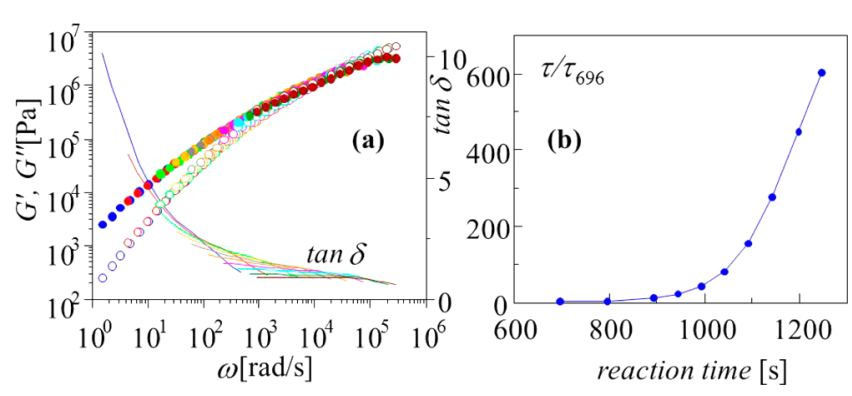

Figure 2. Shifting attempt of the dynamic moduli of DeRosa et al. ${ }^{34,35}$ (a) All data of Figure $1 \mathrm{~b}$ are shifted onto the earliest $G^{\prime}, G^{\prime \prime}$ data (lowest moduli corresponding to highest $2 \delta / \pi$ curve of Figure $1 \mathrm{~b}$ ) in order to show the relative growth of the relaxation times (b).

colloidal suspensions, the fast $\beta$ relaxation is attributed to the localized motion of particles inside "cages" that are formed by their neighboring particles. The slow $\alpha$ relaxation expresses the opening of particle cages and the cooperative dynamics of the particle surroundings. The dynamics becomes spatially heterogeneous due to the varying size of cooperatively moving particle assemblies. ${ }^{41-45}$ The crossover from $\alpha$ to $\beta$ regions results in a pronounced minimum of $G^{\prime \prime}$. This minimum shifts to lower frequencies when the distance to the glass gets small and is often used to estimate the divergence of the longest relaxation time when it begins to diverge to values outside the experimental range.

The rheology of colloidal suspensions has been studied extensively but very few complete data sets are available for determining $G(t)$ or $H(\lambda)$ in the approach of LSTLS, mostly from the groups of Petekidis ${ }^{46}$ and Ballauff. ${ }^{47-51}$ For this study we focus on Siebenbürger's data ${ }^{51}$ because her particles are of tunable size, her data cover a wide frequency range, and her measurements are in close agreement with mode coupling theory (MCT) predictions. Her thermosensitive colloidal suspension is a model system for which tunability was achieved by coating spherical polystyrene particles (diameter $\sim 100 \mathrm{~nm}$ ) with a cross-linked shell of thermosensitive poly(Nisopropylacrylamide) (PNIPAM) and suspending the 
particles in water.$^{47-50}$ Siebenbürger et al. ${ }^{51}$ established stable conditions at constant $\varepsilon_{i}$ closer and closer to LSTLS and measured the evolving rheological properties. The data as shown in Figure 3 suggest a powerlaw relaxation time spectrum
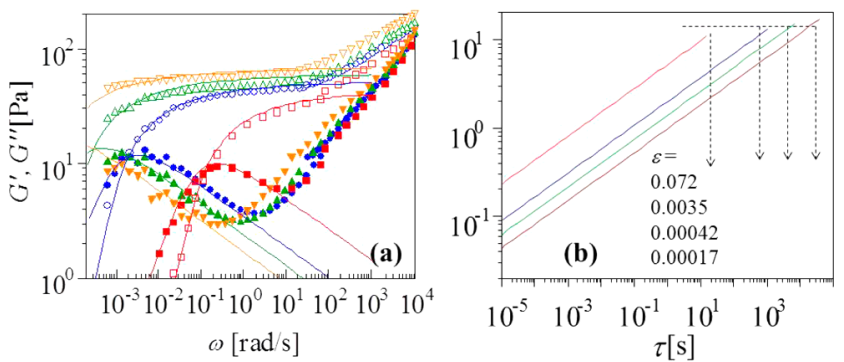

Figure 3. Colloidal glass transition data of Siebenbürger et al. ${ }^{51}$ (a) $G^{\prime}(\omega)$ (open symbols) and $G^{\prime \prime}(\omega)$ (filled symbols) at increasing volume fraction of solids (shifting to the left when decreasing $\varepsilon$ ). While a more detailed rheological analysis is given by Winter et al., ${ }^{52}$ data is matched here with a powerlaw spectrum for the $\alpha$-transition (excluding the discussion of the $\beta$-relaxation) since this study focuses on the long-time dynamics. (b) Powerlaw spectra for the $\alpha$-transition in part a. In the approach of the glass, the entire $\alpha$-spectrum shifts to larger $\tau$-values while maintaining its slope and its highest value. The shifting cutoff of the spectrum expresses the growth of the longest relaxation time, $\tau_{\alpha}$.

in which $\alpha$ - and $\beta$-relaxation processes are considered to be additive, $^{52}$

$$
\begin{aligned}
& H(\tau, \varepsilon)=n_{\alpha} G_{c}\left\{\left(\frac{\tau}{\tau_{\alpha}(\varepsilon)}\right)^{n_{\alpha}}+\left(\frac{\tau}{\tau_{0}}\right)^{-n_{\beta}}\right\}, \\
& \text { for } \tau<\tau_{a}(\varepsilon) \text { and } \varepsilon>0
\end{aligned}
$$

The spectrum is remarkable in its simplicity since it does not introduce any new parameter beyond the known parameters of MCT. No new parameter was added and none was removed. $n_{\alpha}$ and $n_{\beta}$ are positive-valued exponents that originate from MCT. The powerlaw differs significantly from previous models of near-glass dynamics but it expresses the $G^{\prime}, G^{\prime \prime}(\omega)$ data in great detail. Equation 8, when inserted into eqs 1 and 2, becomes a rheological constitutive equation. Equation 8 is otherwise known as the BSW spectrum. ${ }^{53}$ Its properties have been mapped extensively. ${ }^{54,55}$

When getting close to LSTLS, $\varepsilon>0$, the $\alpha$-relaxation modes overpower the shorter relaxation modes. Contributions from the $\beta$-relaxation become negligible. This suggests the approximate relaxation time distribution

$$
H(\tau, \varepsilon)=n_{\alpha} G_{c}\left(\frac{\tau}{\tau_{\alpha}(\varepsilon)}\right)^{n_{\alpha}}, \quad \text { for } \tau<\tau_{a}(\varepsilon) \text { and } n_{\alpha} \geq 0
$$

which is the inverse of the gelation spectrum. $G_{\mathrm{c}}$ is the plateau modulus of the $G^{\prime}$ data.

The powerlaw relaxation dynamics of colloidal glasses, eq 9, can also be found with molecular glass formers, which rely on molecular diffusion for their liquid properties and undergo a glass transition upon lowering the temperature below a critical level. As the glass transition is approached, $T \rightarrow T_{g}$, large-scale molecular motion slows down and dynamics become spatially heterogeneous, which leads to a broad distribution of relaxation processes. $^{56}$ In Figures 4 and 5, the powerlaw behavior of colloidal glasses gets tested with SAOS data of $\mathrm{Xu}$ et $\mathrm{al}^{57}$ for
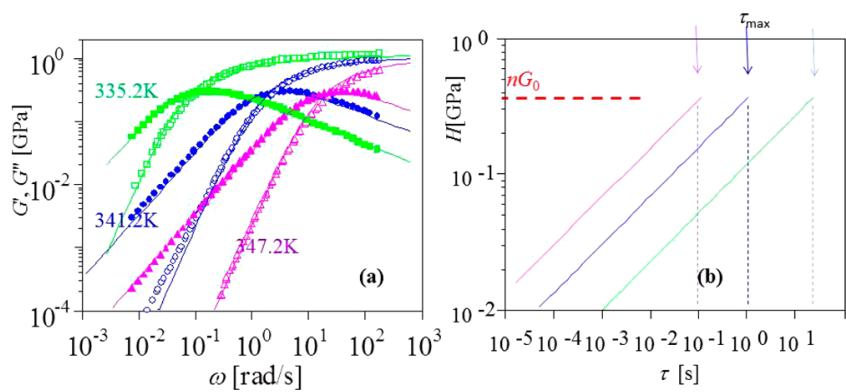

Figure 4. Dynamic modulus data of $\mathrm{Xu}$ and $\mathrm{McKenna}{ }^{57}$ for sucrose benzoate. (a) $\alpha$-Relaxation fully dominates the dynamics in the given frequency range. (b) Same powerlaw spectrum represents all data, except for a shift on the time axis. $H_{0}=n G_{\mathrm{e}}=2.7 \times 10^{8} \mathrm{~Pa}$ and $n=$ 0.33 .
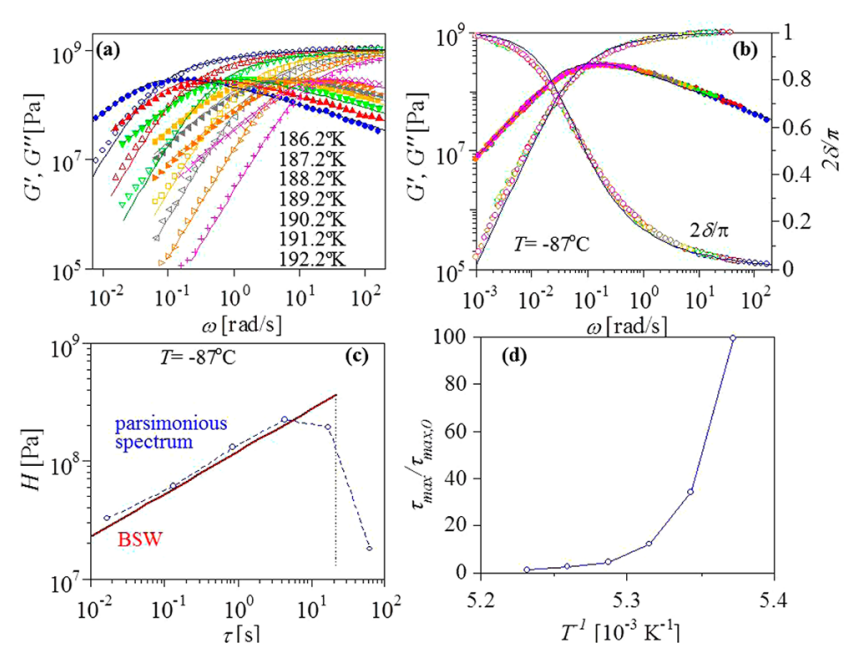

Figure 5. Dynamic modulus data of $\mathrm{Xu}$ and McKenna ${ }^{57}$ for $\mathrm{m}$ toluidine. The $\alpha$-relaxation (a) fully dominates the dynamics in the given frequency range. The moduli shift to the left when lowering the temperature. The data could be collapsed into a single curve (b) and expressed in a relaxation time spectrum (c) with $H_{0}=n G_{c}=3.7 \times 10^{8}$ $\mathrm{Pa}$ and $n=0.36$.The abrupt cutoff of the relaxation time spectrum assumes a very simple format, maybe too simple. Part $\mathrm{c}$ also compares the parsimonious spectrum, ${ }^{17,18}$ dashed line, with the BSW powerlaw spectrum and its sudden cutoff, solid line. The horizontal shift for collapsing the data of part a to the single curve in part $b$ determines the growth of the relaxation time shown in part $\mathrm{d}$.

two molecular glass formers near LSTLS. The data follow the powerlaw relaxation behavior, eq 9, very closely. This agreement further supports the notion that colloidal suspensions are valuable model systems for the study of the molecular glass transition dynamics.

Glass forming materials have been reported to exhibit stretched exponential relaxation dynamics (KWW), ${ }^{58,59}$ an idea that goes back to Kohlrausch ${ }^{60}$ and Williams and Watts ${ }^{61}$ and received its name from there. The KWW relaxation modulus is expressed as

$$
G(t)=g \mathrm{e}^{-(t / \tau)^{n}}
$$

Montroll and Bendler analyzed the properties of $H(\tau)$ that belongs to KWW. ${ }^{62}$ BSW fits the DeRosa data more closely than KWW, but BSW and KWW are surprisingly close to one another. The distribution of KWW and BSW relaxation modes is similar at higher exponent values, $0.6<n<1$, but KWW would be much more broadly distributed than BSW at low 
exponent values. The $\alpha$-transition of DeRosa's polymer is narrower than expressed with KWW but probably not as sharp as shown with BSW and its sudden cutoff. Figure 5c addresses the broadening of the cutoff by comparing the continuous relaxation time spectrum as calculated with the parsimonious model $^{17,18}$ with BSW. ${ }^{53}$ The parsimonious $H(\tau)$ clearly shows the powerlaw (discrete points on a straight line at about equal distance between them) but then combines it with the broadened cutoff.

Divergence of Zero Shear Rate Viscosity. Knowledge of the spectrum allows to calculate the whole range of linear viscoelastic material functions. An example is given here with the longest relaxation time, $\tau_{\max }$, and its relation to the zero shear rate viscosity, $\eta_{0}$. Both diverge in the approach of LSTLS. The known relaxation time spectrum makes it now possible to connect these two material functions. The zero shear rate viscosity is defined as ${ }^{16}$

$$
\begin{aligned}
\eta_{0} & =\int_{0}^{\infty} \mathrm{d} t G(t)=\int_{0}^{\infty} \mathrm{d} t\left(G_{e}+\int_{0}^{\tau_{\max }} \frac{\mathrm{d} \tau}{\tau} H(\tau) \mathrm{e}^{-t / \tau}\right) \\
& =\int_{0}^{\tau_{\max }} \mathrm{d} \tau H(\tau)
\end{aligned}
$$

When only using the dominant part of the relaxation time spectrum, having powerlaw format for gelation and the glass transition, $H=H_{0}\left(\tau / \tau_{\max }\right)^{n}$, the above equation consolidates to

$$
\int_{0}^{\tau_{\max }} \mathrm{d} \tau H_{0}\left(\frac{\tau}{\tau_{\max }}\right)^{n}=\frac{H_{0} \tau_{\max }}{n+1} \text { or } \frac{\eta_{0}}{\tau_{\max }}=\frac{H_{0}}{n+1}
$$

The ratio of viscosity and longest relaxation time turns out to be constant in the approach of the transition from the liquid side. This is a meaningful result, which helps to connect with existing knowledge about gels and glasses.

The diverging viscosity in the approach of the gel point from the liquid side has drawn early attention because of its dramatic appearance. In the close vicinity of the chemical gel point of a cross-linking polymer, the viscosity is predicted to follow a powerlaw, $^{63}$

$$
\eta_{0} \approx \varepsilon^{s}
$$

with the distance from the critical reaction conversion, $\varepsilon=p_{c}-p$, as independent variable.

For the colloidal glass, the diverging relaxation time as predicted by the mode coupling theory ${ }^{64,65}$ allows a prediction now for the diverging zero shear rate viscosity:

$$
\eta_{0}=\frac{n_{\alpha}}{1+n_{\alpha}} G_{c} \tau_{\max }=\frac{n_{\alpha}}{1+n_{\alpha}} G_{c} \tau_{0, \alpha}(\varepsilon)^{-\gamma}
$$

The characteristic relaxation times for the $\alpha$-relaxation is predicted to diverge in a powerlaw in the approach of the glass (for $\varepsilon>0$ ), $\tau_{\alpha}=\tau_{0, \alpha}(\varepsilon)^{-\gamma}$. The divergence of the longest relaxation time and its relation to structural evolution has been studied extensively, see Marcotte et al. ${ }^{15}$ and references therein.

\section{DISCUSSION}

Small amplitude oscillatory shear (SAOS) data are an expression of the dynamics at different structural length scales of gels and glasses in the approach of LSTLS. The glass/gel inverse relaxation property (Figure 6) shows the distinct difference between the two material classes. The findings of this study become plausible when focusing on the slowest mode (longest relaxation time) and its probability to contribute to the

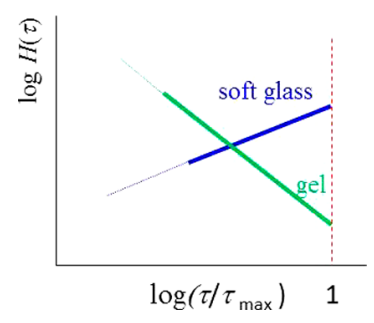

Figure 6. Glass transition spectrum as inverse of relaxation spectrum for gelation.

stress, eq 1. For gels, the slowest relaxation mode is weakest while for glasses the slowest mode dominates as will be discussed next.

For gelation, the characteristic molecular size distribution is assumed to be the main reason for the decaying $H(\tau)$, i.e. the negative exponent of the powerlaw. The molecular weight distribution broadens in the approach of LSTLS and assumes powerlaw format and a negative exponent. ${ }^{66,67}$ The majority of the molecules are still small when the material reaches the gel point and the stress is dominated by rearrangement of the small molecules. The largest molecular cluster diverges in size but has the lowest probability. On the basis of that information, the longest mode can be expected to be weakest since the percolating structure is barely connected. Fast relaxation processes of the low molecular weight fraction dominate the relaxation time spectrum. The resulting spectrum is a decaying function in $\tau$; i.e., the exponent for eq 3 is negative.

Branching is also known to lead to powerlaw relaxation behavior. A gel-like spectrum is found for multibranched polymers which exhibit a broad powerlaw transition from the elastic behavior at intermediate frequencies to the terminal behavior at low frequencies. ${ }^{70}$ The width of the powerlaw region of branched systems depends on temperature, i.e. not satisfying the time-temperature superposition any more. Branching by itself is able to produce powerlaw dynamics as shown for the hypothetical case a polymer with monodisperse, self-similarly branched architecture; ${ }^{68,69}$ this power-law behavior results from the validity of the coarse-graining of the chain structure according to the time scale and from the entropic nature of the stress. The powerlaw shape of $H(\tau)$ of actual gelling systems might be caused by both, the branching distribution and the powerlaw distribution of molecular sizes. Exploring this interrelation is outside the scope of the current study.

The study here concerns gels with sufficiently stable connectivity once an internal bond was formed. This allows the longest relaxation time to fully diverge in the approach of LSTLS. Extension of the study to systems with an upper limit for the diverging $\tau_{\max }$ is considered in the future. The constraint for the growth of longest relaxation time is important for the understanding of many physical gels ${ }^{10}$ and of chemical gelation with reversible bond kinetics. ${ }^{71}$ The presence of an upper limiting relaxation time is less apparent in the approach of the LSTLS but becomes important beyond the gel point where a more and more pronounced $G^{\prime \prime}$ minimum develops as an indication of $\tau_{\max }$ even if $\tau_{\max }$ might be outside the accessible experimental range. The dynamics of such gels in their "solid state" is a large topic by itself and is not addressed here.

For the approach of the colloidal glass transition, the suspended particles can still move freely at low solids loading and relaxation processes in the suspension decay quickly to 
zero. Upon increasing the solids fraction (by expanding the particle size in Siebenbuerger's experiments), the motion of a particle gets more and more impeded by neighboring particles, which behave as cage-forming obstacles. The dynamics undergoes a qualitative change from rather independent to a collective one, in which individual particles can only move when their neighbors open up free space. The system is able to store elastic energy and becomes viscoelastic. Structural rearrangements begin to take longer times. When further upping the particle loading, particle movements become more sluggish, and the characteristic time scales for relaxation processes grow significantly. The motion of an individual particle not only involves particles in their immediate neighborhood but also particles in their wider and wider surrounding. The correlation length of particle motion grows and eventually, when reaching the glass state, diverges to sample size. A nearly complete dynamical arrest sets in when arriving at the glass. The current study is restricted to solid loadings below the critical value for forming the glass.

The powerlaw relaxation spectrum with positive exponent suggests not only that the particle dynamics is heterogeneous in a broad distribution but also that the slow relaxation modes dominate. The powerlaw spectrum with positive exponent is an expression of the high probability of large-scale cooperative motions. Few particles can still move easily by themselves while most others involve the cooperative motion of many particles in their wider surrounding. Unclear is the issue of the sharp cutoff of the spectrum which might be an artifact of the experiment.

It should be noted that the short-range nature of the interparticle force (due to quasi hard-core interaction) seems to be essential for the power-law spectrum. In comparison, charged particles exhibiting long-ranged repulsion do not seem to exhibit the preglass behavior (power-law spectrum) discussed in this manuscript. ${ }^{72}$ Some systems even exhibit rather sharp transitions into colloidal crystal even if the particles are polydisperse in their size and charge.

Similar structural dynamics can be envisioned for the molecular glass formers. Caging might not be the appropriate process. More likely is a distributed relaxation in which few molecules can rearrange by themselves or involve small groups of molecules while most relaxation processes require large-scale cooperation of molecular groups. This again will result in a $H(\tau)$, in which the slow modes overpower the fast ones as expressed in the powerlaw with positive exponent. The agreement with the behavior of colloidal glass formers confirms the understanding that colloidal glasses and molecular glasses share many of their properties.

Noteworthy is the wide $\varepsilon$-range near LSTLS in which the same powerlaw governs the glass transition, at least for the tested materials of this study (most pronounced with the molecular glass formers): the same slope and the same upper end point of $H(\tau)$. The only changes occur in the diverging $\alpha$ relaxation time, the longest relaxation time $\tau_{\max }=\tau_{\alpha}$. In comparison, gelation dynamics follows the powerlaw only in a very narrow $\varepsilon$-range near LSTLS for many materials; unclear is the steadiness of the value of the relaxation exponent which seems to be slowly decaying while passing through the gel point. $^{27,73,74}$ However, Adolf and Martin, ${ }^{36}$ Trappe and Weitz, ${ }^{75}$ and Larsen and Furst ${ }^{76}$ demonstrated how gelation data can be collapsed into a single set of master curves, which shows that for some materials the same relaxation pattern prevails in a wider vicinity of the gel point.
The distinguishing between materials with positive or negative $n$ leads to the question of the physical meaning of the material in between, with $n=0$, and its rheological consequences. Equation 2 together with constant $H\left(\tau, \varepsilon_{i}\right)=H_{0}$ results in an incomplete gamma function for the modulus. The dynamic moduli assume an admissible format,

$$
\begin{gathered}
\frac{G^{\prime}(\omega)}{H_{0}}=\int_{0}^{\omega \tau_{\max }} \frac{x \mathrm{~d} x}{1+x^{2}}=\frac{1}{2} \ln \left(1+\left(\omega \tau_{\max }\right)^{2}\right) ; \\
\frac{G^{\prime \prime}(\omega)}{H_{0}}=\int_{0}^{\omega \tau_{\max }} \frac{\mathrm{d} x}{1+x^{2}}=H_{0} \tan ^{-1}\left(\omega \tau_{\max }\right)
\end{gathered}
$$

but no such material seems to exist. The $n=0$ limit might possibly belong to BSW since it does not show the powerlaw features of gels.

It should be noted that there are other material structures that also lead to powerlaw relaxation. The powerlaw spectra are not unique to the glass transition or the gelation. For instance, molten polymers and polymer solutions with highly entangled, linear, flexible molecules of about uniform length also exhibit BSW dynamics much above the glass transition. ${ }^{77,78}$ The long linear molecules have been viewed as being caged in a narrow linear space ("tube") which preferably allows linear diffusion ("reptation"). 79 The longest relaxation time of the powerlaw substantially grows when increasing molecular weight. In contrast to that, small macromolecules below entanglement molecular weight are governed by fully screened or unscreened hydrodynamic interaction; they relax in a powerlaw spectrum with negative exponent $(n=-1 / 2$ and $-2 / 3$, respectively) and a cutoff at their longest relaxation time; ${ }^{80,81}$ their powerlaw relaxation time spectrum looks very much alike the spectrum of a physical gel or a chemically gelling system that has not yet reached the gel point. $^{22}$

\section{SUMMARY}

Boltzmann with his generally valid equation of linear viscoelasticity sets the stage for this study by having shown that the relaxation time spectrum $H(\tau)$ is the only material function needed for a complete definition of a viscoelastic pattern. Two entirely different relaxation patterns, one for the glass transition and a kind of opposite one for gelation, govern the approach of the liquid-to-solid transition from the liquid side (LSTLS). The spectrum data of a variety of gels and glasses are found to consistently reduce into a powerlaw format, in which gels adopt a negative and glasses a positive powerlaw exponent. The finding is empirical for gelation and for molecular glasses but has theoretical backing through the mode coupling theory for colloidal glasses. The glass/gel inverse property will need to be tested on additional materials and its origin still needs to be explained from first principles. It also will be interesting to see how the two relaxation patterns might interfere with each other in materials in which gelation competes with the glass transition near LSTLS. Also, it is not clear how representative the two model materials are, whether these are the only two rheological patterns for the liquid-tosolid transition of amorphous materials, or whether there are more.

\section{AUTHOR INFORMATION}

\section{Corresponding Author}

*E-mail: winter@ecs.umass.edu. 


\section{Notes}

The authors declare no competing financial interest.

\section{ACKNOWLEDGMENTS}

H.H.W. gratefully acknowledges the support of the NSF which allowed him to continue research while serving as a program director during the past three years. Acknowledgment is made to the donors of the Petroleum Research Fund, administered by the American Chemical Society, for partial support of this research. Many thanks go to Miriam Siebenbürger, Matthias Ballauff, and Matthias Fuchs for sharing their data and for introducing me to colloidal dynamics, experimentally and theoretically, to Ben $\mathrm{Xu}$ and Greg McKenna for sharing their extensive modulus data, and to Jeffrey Morris, Dimitris Vlassopoulos, and Hiroshi Watanabe for helpful discussions. All data analysis and plotting was done with the Interactive Rheology Information Software (IRIS). ${ }^{82}$

\section{REFERENCES}

(1) Mason, T. G.; Weitz, D. A. Phys. Rev. Lett. 1995, 75, 2770-2773.

(2) Parker, A.; Normand, V. Soft Matter 2010, 6, 4916-4919.

(3) Larson, R. G. The Structure and Rheology of Complex Fluids. Oxford Univ Press: New York, 1999.

(4) Zaccarelli, E.; Poon, W. C. K. Proc. Natl. Acad. Sci. U.S.A. 2009, 106, 15203-15208.

(5) Jabbari-Farouji, S.; Wegdam, G. H.; Bonn, D. Phys. Rev. Lett. 2007, 99, 065701.

(6) Eckert, T.; Bartsch, E. Phys. Rev. Lett. 2002, 89, 125701.

(7) Willenbacher, N.; Vesaratchanon, J. S.; Thorwarth, O.; Bartsch, E. Soft Matter 2011, 7, 5777-88.

(8) Shibayama, M.; Ozeki, S.; Norisuye, T. Polymer 2005, 46, 238188 .

(9) Boltzmann, L. Sitzungsber. Kaiserl. Akad. Wiss. Wien, Math.Naturwiss. 1874, 70, 275-306.

(10) te Nijenhuis, K. Adv. Polym. Sci. 1997, 130, 1-267.

(11) Hunter, G. L.; Weeks, E. R. Rep. Prog. Phys. 2012, 75, 066501.

(12) Cheng, Z.; Zhu, J.; Chaikin, P. M.; Phan, S. E.; Russel, W. B. Phys. Rev. E 2002, 65, 041405.

(13) Torquato, S.; Truskett, T. M.; Debenedetti, P. G. Phys. Rev. Lett. 2000, 84, 2064

(14) Bernal, J. D. Proc. R. Soc. London A 1964, 280, 299.

(15) Marcotte, E.; Stillinger, F. H.; Torquato, S. J. Chem. Phys. 2013, $138,12 \mathrm{~A} 508$.

(16) Ferry, J. D. Viscoelastic Properties of Polymers, 3rd. ed.; Wiley: New York, 1980.

(17) Baumgärtel, M.; Winter, H. H. Rheol. Acta 1989, 28, 511-519.

(18) Baumgärtel, M.; Winter, H. H. J Non-Newtonian Fluid Mech. 1992, 44, 15-36.

(19) Mours, M.; Winter, H. H. Rheol. Acta 1994, 33, 385-397.

(20) Holly, E. E.; Venkataraman, S. K.; Chambon, F.; Winter, H. H. J

Non-Newtonian Fluid Mech. 1988, 27, 17-26.

(21) Ghiringhelli, E.; Roux, D.; Bleses, D.; Galliard, H.; Caton, F. Rheol. Acta 2012, 51, 413-420.

(22) Chambon, F.; Winter, H. H. Polym. Bull. 1985, 13, 499-503.

(23) Winter, H. H., Chambon, F. Rheology of crosslinking polymers at the gel point; Proceedings of the Bi-annual Meeting; Polymer Networks Group: Elsinore, Denmark, 1986.

(24) Winter, H. H.; Chambon, F. J. Rheol. 1986, 30, 367-382.

(25) Chambon, F.; Winter, H. H. J. Rheol. 1987, 31, 683-697.

(26) Izuka, A.; Winter, H. H.; Hashimoto, T. Macromol 1994, 27, 6883-6888.

(27) Friedrich, C.; Heymann, L.; Berger, H. R. Rheol. Acta 1989, 28, 535-539.

(28) Morris, E. R.; Nishinari, K.; Marguerite, R. Food Hydrocolloids 2012, 28, 373-411.

(29) Aliaghaie, M.; Mirzadeh, H.; Dashtimoghadam, E.; Taranejoo, S. Soft Matter 2012, 8, 3128-3137.
(30) Aoki, Y. Rheol. Acta 2011, 50, 787-793.

(31) Bonino, C. A.; Samorezov, J. E.; Jeon, O.; Alsberg, E.; Khan, S. A. Soft Matter 2011, 7, 11510-11517.

(32) Ng, T. S. K.; McKinley, G. H.; Ewoldt, R. H. J. Rheol. 2011, 55, 627-654.

(33) Eberle, A. P. R.; Wagner, N. J.; Castaneda-Priego, R. Phys. Rev. Lett. 2011, 106, 105704.

(34) DeRosa, M. E.; Winter, H. H. Rheol. Acta 1994, 33, 220-237.

(35) DeRosa, M. E.; Mours, M.; Winter, H. H. Polym. Gels Networks 1997, 5, 69-94.

(36) Adolf, D.; Martin, J. E. Macromol 1990, 23, 3700-3704.

(37) van Megen, W.; Pusey, P. N. Phys Rev A 1991, 43, 5429.

(38) Sciortino, F.; Tartaglia, P. Adv. Phys. 2005, 54, 471.

(39) Pusey, P. N. J. Phys.: Condens. Matter 2008, 20, 494202.

(40) Pham, K. N.; Petekidis, G.; Vlassopoulos, D.; Egelhaaf, S. U.; Poon, W. C. K.; Pusey, P. N. J. Rheol. 2008, 52, 649-676.

(41) Habdas, P.; Weeks, E. R. Curr. Opin. Colloid Interface Sci. 2002, 7, 196-203.

(42) Prasad, V.; Semwogerere, D.; Weeks, E. R. J. Phys: Condens Matter 2007, 19, 113102.

(43) Narumi, T.; Franklin, S. V.; Desmond, K. W.; Tokuyama, M.; Weeks, E. R. Soft Matter 2011, 7, 1472.

(44) Ballesta, P.; Duri, A.; Cipelletti, L. Nature Phys. 2008, 4, 550.

(45) Sessoms, D. A.; Bischofberger, I.; Cipelletti, L.; Trappe, V. Philos. Trans. R. Soc. A 2009, 367, 5013.

(46) Koumakis, N.; Pamvouxoglou, A.; Poulosa, A. S.; Petekidis, G. Soft Matter 2012, 8, 4271-82.

(47) Crassous, J. J.; Siebenbürger, M.; Ballauff, M.; Drechsler, M.; Hajnal, D.; Henrich, O.; Fuchs, M. J. Chem. Phys. 2006, 125, 204906.

(48) Crassous, J. J.; Ballauff, M.; Drechsler, M.; Schmidt, J.; Talmon, Y. Langmuir 2006, 22, 2403.

(49) Crassous, J. J.; Siebenbürger, M.; Ballauff, M.; Drechsler, M.; Hajnal, D.; Henrich, O.; Fuchs, M. J. Chem. Phys. 2008, 128, 204902.

(50) Crassous, J. J.; Wittemann, A.; Siebenbürger, M.; Schrinner, M.; Drechsler, M.; Ballauff, M. Colloid Polym. Sci. 2008, 286, 805.

(51) Siebenbürger, M.; Fuchs, M.; Winter, H. H.; Ballauff, M. J. Rheol. 2009, 53, 707-720.

(52) Winter, H. H.; Siebenbürger, M.; Hajnal, D.; Henrich, O.; Fuchs, M.; Ballauff, M. Rheol. Acta 2009, 48, 747-753.

(53) Baumgärtel, M.; Schausberger, A.; Winter, H. H. Rheol. Acta 1990, 29, 400-408.

(54) Carri, G.; Winter, H. H. Rheol. Acta 1997, 36, 330-344.

(55) Friedrich, C.; Waizenegger, F.; Winter, H. H. Rheol. Acta 2007, 47 (8), 909-916.

(56) Ediger, M. Annu. Rev. Phys. Chem. 2000, 51, 99-128.

(57) Xu, B.; McKenna, G. B. J. Chem. Phys. 2011, 134, 124902-7.

(58) Berry, G. C.; Plazek, D. J. Rheol. Acta 1997, 36, 320-329.

(59) Ngai, K. L.; Plazek, D. J.; Rendell, R. W. Rheol. Acta 1997, 36, 307-319.

(60) Kohlrausch, R. H. A. Poggendorff 1854, 91 (56-82), 179-213. (61) Williams, G.; Watts, D. C. Trans Faraday Soc 1970, 66, 80-85.

(62) Montroll, E. W.; Bendler, T. J. J. Stat. Phys. 1984, 34, 129-162.

(63) Stauffer, D.; Coniglio, A.; Adam, A. Adv. Polym. Sci. 1982, 44, 103.

(64) Götze, W. In Liquids, Freezing and Glass Transition, Hansen, J. P., Levesque, D., Zinn-Justin, J.; Eds.; Session LI of Les Houches Summer Schools of Theoretical Physics; North-Holland: Amsterdam, 1991; Vol. 287.

(65) Götze, W.; Sjögren, L. Rep. Prog. Phys. 1992, 55, 241.

(66) Schosseler, S.; Leibler, L. Phys. Lett 1984, 45, 501-507.

(67) Martin, J. E.; Adolf, D.; Wilcoxon, J. P. Phys. Rev. Lett. 1988, 61, $2620-2623$.

(68) Cates, M. E. Phys. Rev. Lett. 1984, 53, 926-929.

(69) Muthukumar, M. J. Chem. Phys. 1985, 83, 3161-3168.

(70) Robertson, C. G.; Garcia-Franco, C. A.; Srinivas, S. J. Polym. Sci., Part B: Polym. Phys. 2004, 42, 1671-1684.

(71) Wang, M., Roth, M., Winter, H. H., Auernhammer, G. K. Mechanical properties and structure in precipitated colloidal silica; XVIth Int Congr Rheology, Book Abstr., 2012; p169 
(72) Fagan, M. E.; Zukoski, C. F. J. Rheol. 1997, 41, 373-397.

(73) Mours, M.; Winter, H. H. Macromol 1996, 29, 7221-7229.

(74) Winter, H. H.; Mours, M. Adv. Polym. Sci. 1997, 134, 165-234.

(75) Trappe, V.; Weitz, D. A. Phys. Rev. Lett. 2000, 85, 449-452.

(76) Larsen, T. H.; Furst, E. M. Phys. Rev. Lett. 2008, 100, 146001.

(77) Abdel-Goad, M.; Pyckhout-Hintzen, W.; Kahle, S.; Allgaier, J.;

Richter, D.; Fetters, L. J. Macromolecules 2004, 37, 8135-8144.

(78) Baumgärtel, M.; DeRosa, M. E.; Machado, J.; Masse, M.; Winter, H. H. Rheol. Acta 1992, 31, 75-82.

(79) Doi, M., Edwards, S. F. The Theory of Polymer Dynamics; Oxford U Press: Oxford, U.K., 1988.

(80) Rouse, P. E. J. Chem. Phys. 1953, 21, 1272-1280.

(81) Zimm, B. H. J. Chem. Phys. 1956, 24, 269-278.

(82) Winter, H. H.; Mours, M. Rheol. Acta 2006, 45, 331-338. 